\title{
Slosh Baffle Design and Test for Spherical Liquid Oxygen and Liquid Methane Propellant Tank for a Lander
}

\author{
Alan Strahan \\ NASA Johnson Space Center \\ Humberto Hernandez \\ NASA Johnson Space Center
}

\begin{abstract}
A Vertical Test Bed (VTB) is being developed to investigate exploration technologies with earth-based landing trajectories. During this activity, a concern emerged that the VTB, with large liquid tanks, could experience unstable slosh interaction between the propellant fluid motion and the control system, leading to an investigation of slosh characteristics of the VTB. As such, slosh modeling, analysis and testing were performed, that both verified models and lead to the conclusion that baffles would be required for the full-scale vehicle. Follow-on design and testing supported development of these baffles and measurement of their performance. The majority of the tests conducted, including both subscale and full, involved the use of clear tanks containing water as a reasonable substitute for the cryogenic propellants, though a few tests involved the actual liquid oxygen and methane. Along the way, some unique test and data recording methods were employed to reduce testing complexity and cost.
\end{abstract}

\section{Introduction}

The NASA Johnson Space Center is developing a modest size rocket powered Vertical Test Bed (VTB), entitled Project Morpheus, to support the investigation and development of various exploration and planetary landing related technologies while flying in a more accessible and inexpensive terrestrial environment. Some of these technologies include autonomous surface hazard avoidance sensors, new tank design concepts and the use of alternative propellants, such as liquid oxygen and methane. In addition the project is providing an opportunity for NASA engineers to develop new concepts and follow them through manufacture, testing and flight, fostering skills to support future exploration initiatives.

To support its primary function, the Morpheus VTB must be able to maintain a stable flight attitude, which requires the understanding of its rigid vehicle dynamic response, Center-of-Mass travel and main engine gimbal performance. Additionally, the oscillatory nature, or slosh, of its on-board propellants, along with their potential to interact with flight control, must also be characterized, and if necessary mitigated. This can be a concern as depending on propellant loading, the sloshing mass can account for a significant portion of the total vehicle mass and if its motion is appropriately out-of-phase with the vehicles during a maneuver or disturbance rejection, then the flight control can respond so as to induce and further amplify fluid motion, ultimately leading to a loss of 
control. This is particularly important for a VTB performing flights in the terrestrial environment, where it will often thrust at or above one earth $\mathrm{g}$ (as compared to a much lower thrust during a lunar or asteroid landing), and where it will perform dynamic maneuvers to simulate alternative landing site divert trajectories, all the while operating in the presence of atmospheric winds and gusts.

This paper will present significant slosh investigation activities and results that occurred during the development of the Morpheus VTB in 2010 and 11, including efforts to model, predict and measure slosh in various subscale articles using either water or cryogenic fluids, along with the determination that slosh, unless mitigated, could interact with flight control in an undesired manner. This lead the program to develop and integrate baffles into the full-scale Morpheus tanks, requiring additional design, modeling and testing, an ongoing effort with some results captured here. A secondary goal during this activity, consistent with the Morpheus objective of developing people along with new and original engineering practices, was the exploration of slosh testing methodologies that were simple and, if possible, inexpensive yet with sufficient depth to verify models and address flight worthiness questions.

Ultimately, this work will support the terrestrial flights of the cryogenic VTB, which will in turn test, demonstrate and evolve planetary lander technologies to support human and robotic missions to the moon, Mars, and beyond.

\section{Analytic Slosh Prediction}

To better understand slosh and to support the ability to rapidly characterize slosh performance for different tank shapes and spacecraft configurations, analytic closed form modeling methods were initially employed. This was first pursued in support of lunar lander configuration studies, where analytic slosh prediction compared reasonably well with a more rigorous finite element Computational Fluid Design (CFD) approach [3] for modeling a cylindrical tank with spherical end caps. It should also be noted that CFD slosh modeling is being developed for Project Morpheus, though most work to date still involves an analytic approach.

The Analytic methods employed here generally trace their lineage back to Apollo related work both by NASA and by the Southwest Research Institute (SwRI) in the early to mid 1960's, where substantial number of laboratory experiments and technical documents were captured in 1966 in a single 200+ page NASA treatise on the subject [1]. This documents was repackaged, updated and published again in 2000 by SwRI entitled ' The New "Dynamics Behavior of Liquids in Moving Containers" '. [2]

From these works, analytic slosh modeling techniques were identified and used. The most basic, and one of the most important of these, is the determination of slosh frequency based on a mass-pendulum slosh model. A mass-pendulum model was chosen for this effort over the also common mass-spring model as the mass-pendulum approach more easily adapts to different longitudinal acceleration levels (as provided by a thrusting main engine) without having to recalculate model values, such as the spring constant, and is well suited to simple axial symmetric tanks shapes such as spherical or cylindrical [2].

Frequency is one of the most important slosh characteristics, simply because if it is near the desired flight control frequency (or bandwidth), it has greater potential for undesired interaction [3]. For a spherical or cylindrical tank, slosh frequency, along with 
the fluid participatory mass ratio and pendulum pivot location are simply functions of fill level, tank size and axial (longitudinal) acceleration, where the slosh reaction of most concern typically is the lateral motion of the sloshing mass, perpendicular to the main acceleration [2]. This is applicable for either a thrusting rocket or, appropriate for investigation, a tank suspended in a gravity field. Interestingly enough the fluid type (water, hydrazine, etc.) does not effect any of these characteristics, though the physical sloshing mass (as opposed to its participatory mass ratio) is related to fluid type. This fluid neutrality allows for the convenient use of water in most slosh testing as opposed to the use of difficult-to-manage fluids such as cryogenic liquid oxygen (LOX) or methane. [2]

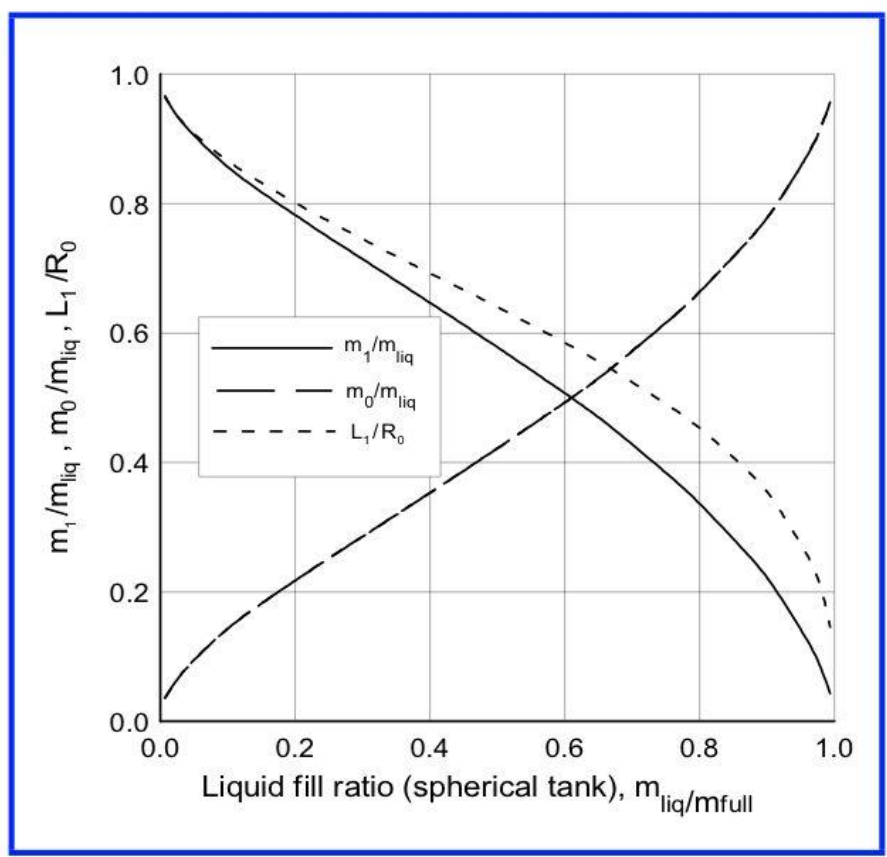

fig-1 slosh performance prediction for spherical tank [2]

$$
f=\frac{1}{2 \pi} \sqrt{\frac{g}{L}}
$$

For the determination of slosh frequency or participatory mass fraction as a function of fill level in a spherical tank, a simple empirically derived curve fit is applied, as represented in fig-1. The $\mathrm{X}$-axis is simply a liquid fill ratio (by volume) while the $\mathrm{Y}$-axis defines 3 different curves where $\mathrm{L}_{1} / \mathrm{R}_{0}$ (small dots) represents the modeled pendulum length vs tank radius, and the $\mathrm{m}_{1} / \mathrm{m}_{\text {liq }}$ the participatory mass ratio. To calculate slosh frequency (in $\mathrm{Hz}$ ) the basic pendulum equation (eq 1) [4] is then used, where length $\mathrm{L}$ is calculated from the pendulum length ratio multiplied by the tank radius, and the effective acceleration $g$ provided as either the main engine acceleration or simply the gravity constant, if the tank is suspended in an earth based lab. For a spherical tank the effective slosh pivot point is simply assumed to be the tank center. For a vertical cylindrical tank, slightly more complicated equations involving hyperbolic trigonometric functions are required, and the pivot point is allowed to travel along the tanks vertical axis, but the computations are still quite simple.

The other analytically derived slosh characteristic of interest for this investigation is the damping ratio of the fluid (which can be augmented by baffles or other devices). The 
damping ratio $\gamma$ for a decaying oscillating profile, commonly used to characterize how rapid the decay, is typically given by the eq- 2 , where $\mathrm{x}_{1}$ and $\mathrm{x}_{2}$ are successive values of the decaying amplitude. Sloshing fluids have their own damping characteristics related to their specific viscosity (effectively internal shear), which does vary substantially with fluid type. [6]

$$
\gamma=\frac{\delta}{\sqrt{(2 \pi)^{2}+\delta^{2}}} \quad \text { where } \quad \delta \triangleq \ln \frac{x_{1}}{x_{2}}
$$

eq-2

To estimate damping of a sloshing fluid, first a dimensionless parameter $\operatorname{Re}_{1}$ (analogous to an inverse Reynolds Number) is estimated, via (eq 3), [2] where $\mathrm{v}$ is the fluids kinematic viscosity, $\mathrm{L}$ a characteristic length such as tank radius, and $\mathrm{g}$ acceleration

$$
\operatorname{Re}_{1}=\frac{\mathrm{v}}{\sqrt{g L^{3}}}
$$

eq-3

due to gravity. The fluid's $\operatorname{Re}_{1}$ value is then used with various equations, depending on tank shape, to determine fluid damping vs fill level. The following two equations are typical methods of estimating a fluids damping in a spherical tank, where (eq 4a) covers fill levels below the mid point, and (eq 4b) above.

$$
\begin{aligned}
& \gamma=0.79 \sqrt{R e_{1}}(R / h) \quad \text { for } \quad 0.1 R \leq h \leq R \\
& \gamma=0.79 \sqrt{R e_{1}}[1+0.46(2-h / R)] /[1.46(2-h / R)] \text { for } h \geq R \quad \text { eq- } 4 \mathrm{~b}
\end{aligned}
$$

Figure 2 shows an important trend in the damping ratio of a spherical tank vs its fill level, in that at the mid point (represented here by $1.0=\mathrm{h} / \mathrm{R}$, or fill height equals radius) the damping is lowest and increases with either lower or higher fills. As such, spacecraft performing maneuvers with a half full tanks are more likely to experience slosh.

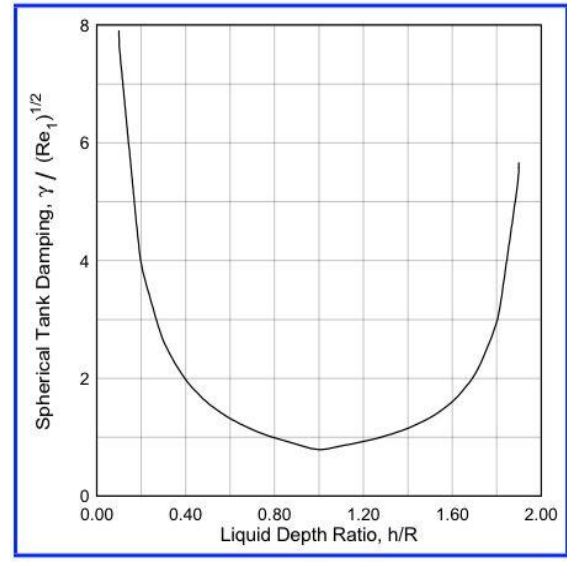

fig-2 damping ratio vs depth ratio for a spherical tank

\begin{tabular}{|c|c|c|c|}
\hline Fill Level & Water & LOX & CH4 \\
\hline $10 \%$ & 0.0013 & 0.00057 & 0.00066 \\
\hline $50 \%$ & 0.00051 & 0.00022 & 0.00026 \\
\hline $80 \%$ & 0.00076 & 0.00033 & 0.00038 \\
\hline
\end{tabular}

table-1 damping ratios for fluids in a spherical tnak 
Finally, table- 1 is included to show the estimated damping ratio () of 3 different fluid types at a low fill level, (10\%) and a mid fill level, for a $4 \mathrm{ft}$ radius spherical tank, demonstrating both how damping reduces at the mid fill, and how damping can vary, with fluid type, in particular that cryogenic propellants are observed to dampen much less than water, also indicating a potential for spacecraft slosh using cryogenic propellants.

\section{Subscale Testing}

In an attempt to physically observe and understand slosh, as well as determine if analytic predictions of slosh frequency could be verified, a simple test set-up involving the suspension of a single transparent spherical tank, was established in a garage (fig 3). The 10-inch glass sphere, purchased at a novelty shop for a few dollars, had an open end for ease of water fills. A net and cargo strap were used along with a hook in the ceiling to suspend the sphere, and food coloring added to aid fluid visibility. A metronome was used to accurately count various frequencies of interest where the sphere would be shaken laterally a small distance (maybe $+/$ - one inch) by hand in time with the metronome. With this, it was observed that excitation at a certain frequencies could easily get the fluid cleanly oscillating back and forth with a generally smooth upper surface, and ringing for several cycles. Excitations above or below this frequency, by $20 \%$ or more would result in less clean oscillations with the upper fluid surface often splashing or otherwise generating several disorganized local waves, typically damping much more quickly. Furthermore the clean oscillating frequency, assumed to be slosh, was observed to be reasonably close to predictions via analytic methods. While this simple activity did not yield results to confirm participatory mass estimates (requiring sensitive load sensors) it did confirm general frequency estimates vs fill level, which are most important, along with damping, in determining the potential for flight control interaction. This garage activity started the development of slosh testing methodologies that have been employed throughout the Morpheus slosh investigation.

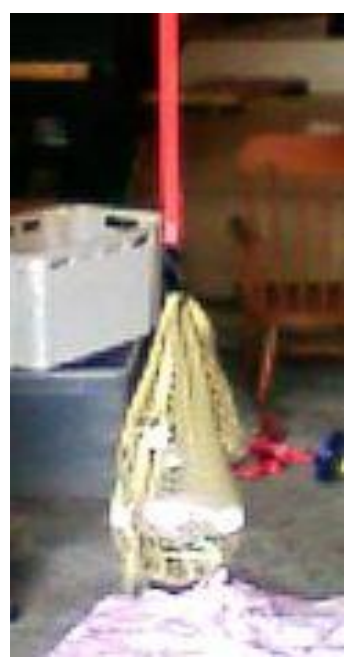

fig-3, garage slosh testing

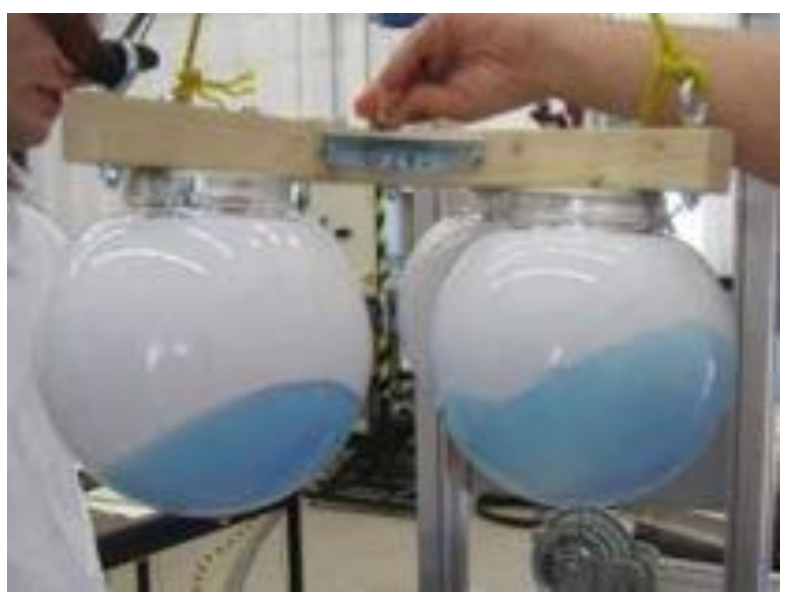

fig-4, small scale cruciform tank cluster 
To explore multi-tank interactions that could potentially present themselves on a Morpheus VTB type lander configuration, a subscale test article with four tanks in a horizontal cruciform configuration was assemble using about 80 dollars in parts from a local Home Depot (fig 4). This test article was suspended in a lab at the Johnson Space Center and subjected to similar type testing as was pursued in the garage with the single tank, where again the test article was laterally excited, by hand, at different frequencies in time with a metronome. Probably the most important observation from this test was that if the 4 tanks were filled to similar levels, the fluid would generally respond similarly to a lateral excitation. After some amount of time and amplitude decay, they may start to diverge a little and trade excitations from one to another though for the most part they behaved similarly for slosh oscillation and damping.

During this testing, excitation methodology was explored as well where lateral inputs appeared to be the most effective at generating a lasting slosh oscillation, though rocking the test article about a lateral axis could produce some slosh response as well. Both techniques would eventually be employed with larger scale testing.

\section{Pixel Testing}

As an intermediate step in their overall engineering development program, the Morpheus Project acquired a mid-size quad tank vertical lander that was no longer in use, from Armadillo Aerospace of Caddo Mills Texas. The mid-sized lander, dubbed 'Pixel' by Armadillo, was of a cruciform layout similar to the envisioned full-scale Morpheus vehicle and was converted to use liquid methane, instead of ethanol, as a fuel to mix with liquid oxygen. Project Morpheus then used the Pixel lander for mounting additional navigation sensors and as an early test bed to gain experience with tethered and free flight operation.

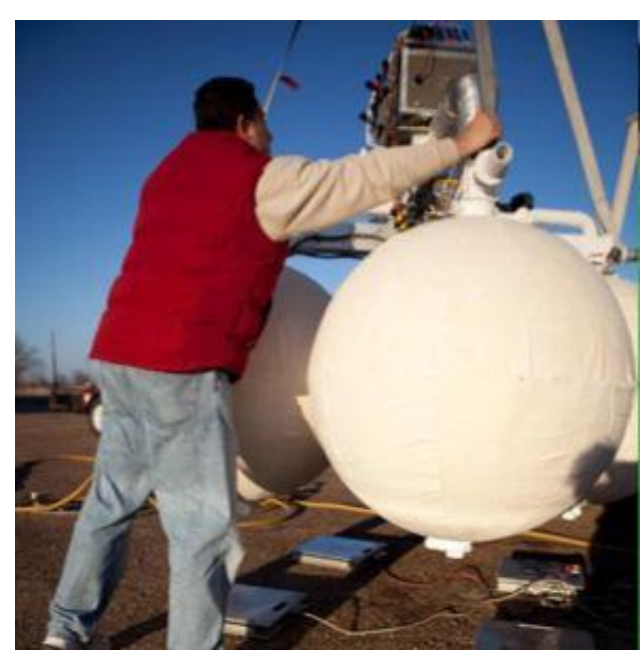

figure-5, Armadillo 'Pixel' test article

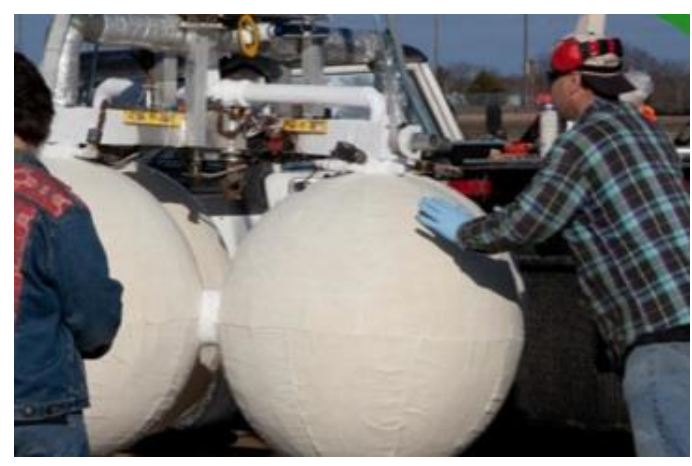

fig 6 - Armadillo 'Pixel' test article

In preparation for both tethered and free flight testing of 'Pixel', an effort was made to model slosh performance, in particular frequency and damping vs fill level, and then to measure such performance with the flight article filled with cryogenic propellants. 
Making this more challenging was that the tanks were opaque, (metal further covered with insulation), with no practical way to insert a camera to observe fluid response. To introduce slosh, subscale excitation methods were again used, with the test article suspended from a crane and one person laterally exciting (shaking) the vehicle $+/-2-3$ inches, in time with a metronome at frequencies predicted to match slosh for the given tank size ( $3 \mathrm{ft}$ diameter each) and for various fill levels. TO address incorrect predictions, excitations were also introduced at frequencies approximately $20 \%$ above and below the target value. During all testing, accelerometer and gyro data from the test article Inertial Measurement Unit (IMU) was recorded. Fig-5 and 6 provide images of this activity.

Fig-7 is an accelerometer trace for both lateral axes for a particular test condition at $45 \%$ full where the red trace is along the primary excitation axis. The X-axis is in milisec and the frequency can be observed to abruptly transition from $1.25 \mathrm{~Hz}$, the excitation frequency for this test, to approximately $1.0 \mathrm{~Hz}$ at $35 \mathrm{sec}$, when the test article was released to oscillate freely. It can also be observed that the cryogenic propellants 'ring' for a considerable amount of time with minimal damping, as expected for cryogenic fluids without baffles. The Armadillo team mentioned that 'Pixel' appeared to have only marginal stability during flight and would oscillate or as one engineer put it "do the hula".

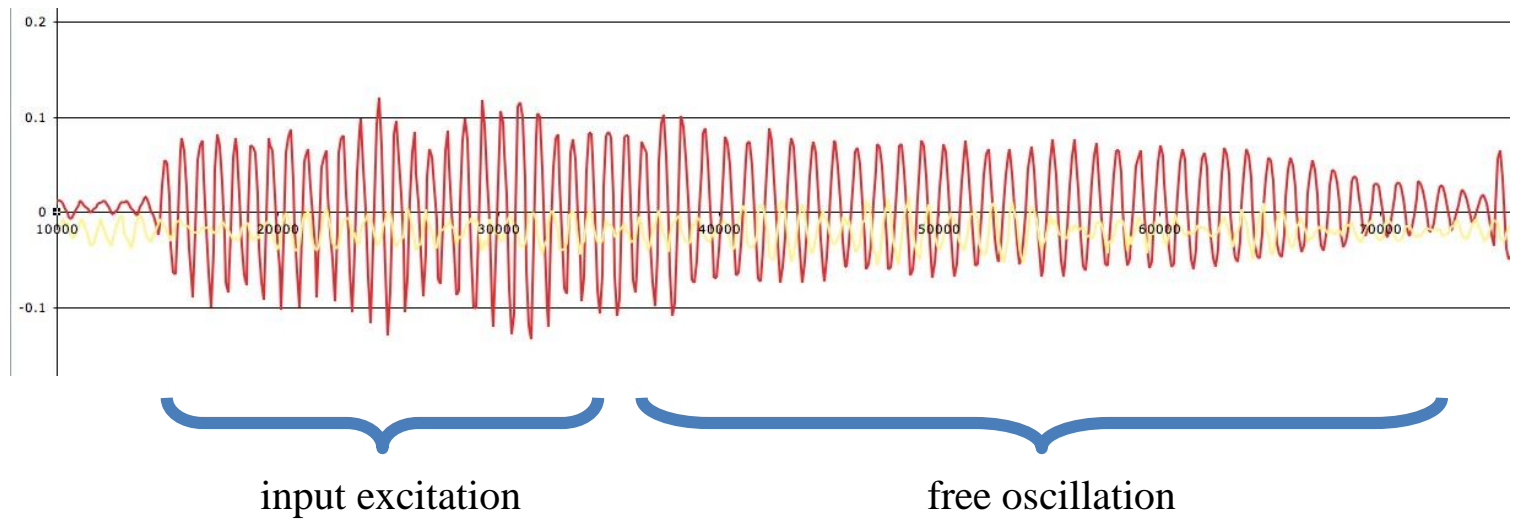

fig-7, lateral oscillations

Table-2 shows predicted and observed slosh performance of the propellants at different flight levels. The predicted frequencies for both fluids is the same, according to theory and at the high and low fill levels appeared to match very close to that observed, while for the mid level, predictions appeared about $10 \%$ slower than observations, which is still reasonable for slosh mitigation assessment and design. The predicted damping is fairly similar, though two different cryogenic propellants are involved, such that for expediency they were tested together ( 2 tanks with methane, two with LOX). In all cases, the predicted damping appeared to show trends similar to that observed, though with values significantly lower, even considering how crude the observed damping estimations were due in part to wave profile interpretation. The observed number of cycles to half amplitude could be off by 5 or 10 , but not by $100+$, which would be necessary to achieve damping as low as predicted. This raises some question with the analytic damping model for clean spherical tanks, which neglected effects such as wall friction and surface 
tension. Such predictions appear to be reasonable for trends but not for the actual magnitude of damping estimation.

\begin{tabular}{|c|c|c|c|c|c|}
\hline Fill level & $\begin{array}{l}\text { Predicted slosh } \\
\text { frequency }(\mathrm{hz})\end{array}$ & $\begin{array}{l}\text { Observed slosh } \\
\text { frequency (hz) }\end{array}$ & $\begin{array}{l}\text { Predicted damp } \\
\text { ratio LOX (\%) }\end{array}$ & $\begin{array}{l}\text { Predicted damp } \\
\text { ratio CH4 (\%) }\end{array}$ & $\begin{array}{l}\text { Observed damp } \\
\text { ratio (\%) }\end{array}$ \\
\hline $90 \%$ & 1.25 & 1.21 & 0.00051 & 0.00059 & 0.0035 \\
\hline $45 \%$ & 0.90 & 1.02 & 0.00026 & 0.00030 & 0.0027 \\
\hline $22 \%$ & 0.83 & 0.86 & 0.00039 & 0.00045 & 0.0040 \\
\hline
\end{tabular}

Table-2, slosh prediction vs observation for Pixel lander

\section{Baffle Design and Test}

As the slosh frequencies from the 'Pixel' testing were observed to be close to prediction and fairly low, approximately one hz and lower, some concern of possible flight control interaction emerged [3]. Though damping is higher than predicted for a clean spherical tank, it is still substantially lower than desired typically to prevent flight control interaction, when slosh frequencies are low [2], [3]. Typically damping ratios of 0.01 to 0.03 are desired for this purpose. Slosh concern was further buoyed by the prediction of even lower slosh frequencies for the larger Morpheus tanks, and with the expectation of the VTB eventually performing aggressive flight maneuvers when it attempted landing target re-designation in response to hazard detection, and flying in winds and gusts. All this lead to the decision to incorporate baffles in the Morpheus tanks.

To design baffles for a spherical tank, an analytic design methodology, empirically derived, was applied from the slosh literature. The following equation (eq-5) was identified from multiple sources in the literature [2], [5]. The equation supports horizontal ring baffle design, which is a common for spherical or cylindrical tanks and reasonably simple to model. This equation originally was for a cylindrical tank but can be applied to a sphere, if the region immediately above and below that where the equation is applied is assumed cylindrical, an assumption that is most limited really only near the top and bottom of the sphere. The equation defines the predicted fluid damping ratio, $\gamma$, due to baffles at a particular fill level above a given baffle (captured by $h_{s}$ over $R$ ), factoring in

$$
\gamma=2.83 e^{-4.6\left(h_{s} / R\right)} C_{1}^{3 / 2}(\delta / R)^{1 / 2}
$$

eq-5

the baffle width (represented by an expansion of the $\mathrm{C}_{1}$ term), and a ratio of desired maximum slosh wave height over tank radius $(\delta / \mathrm{R})$, which itself is assumed not to exceed 0.2. This equation was applied to the design of a 48 inch diameter spherical Morpheus tank via a spread sheet at one inch intervals for heights between 6 and 36 inches ( 6 inches from the top and bottom), covering a range of 5 to $95 \%$ fill volume, with the assumption that sloshing mass above and below this region would be negligible. The design goal was 
to keep the damping ratio above 0.025 , a reasonable goal to minimize sloshing according to the literature [2], [5]. The resulting design damping ratio vs tank height is represented by the saw-tooth curve in fig- 12 below.

Continuing with the strategy to test design concepts and compare to prediction, both a sub-scale and a full-scale test campaign was conducted for the ring-baffle design.

Initially a 12-inch spherical transparent tank was used with and without sub-scale ring baffles, where as before the tank was filled to various levels with water and laterally excited (shaken) in time with a metronome (fig-8). Though not included here, the results showed substantial improvement in fluid damping with the inclusion of the scaled baffle design.

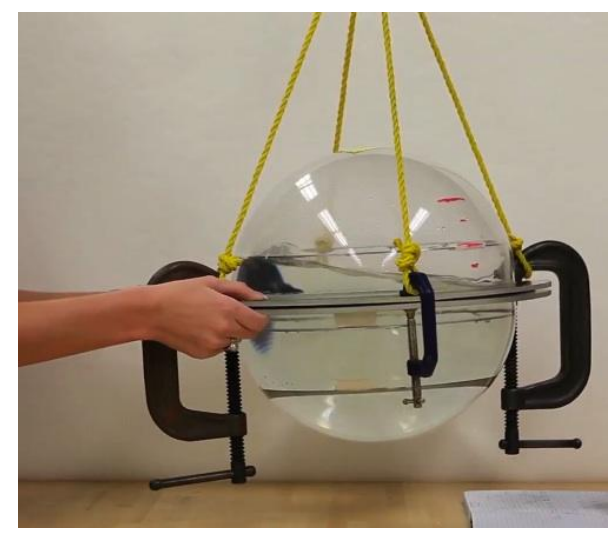

fig-8, subscale slosh baffle testing

Baffle design testing was then performed with a full-scale 48 inch diameter acrylic tank. The acrylic tank was inexpensive, transparent and strong, as it would have to hold up to about one ton of water at its $95 \%$ full test level. As with the subscale sphere, the full-scale consisted of two hemispheres that could be separated for baffle installation, and that were then sealed with clamps and a gasket to prevent leakage. The sphere had a fill hole and drain valve on the top and bottom respectively and was suspended in a high-bay via a crane above rings of sandbags should the suspension inadvertently fail (fig-9). Those conducting the test had to wear strong-toed boots and hard hats as additional precaution. 


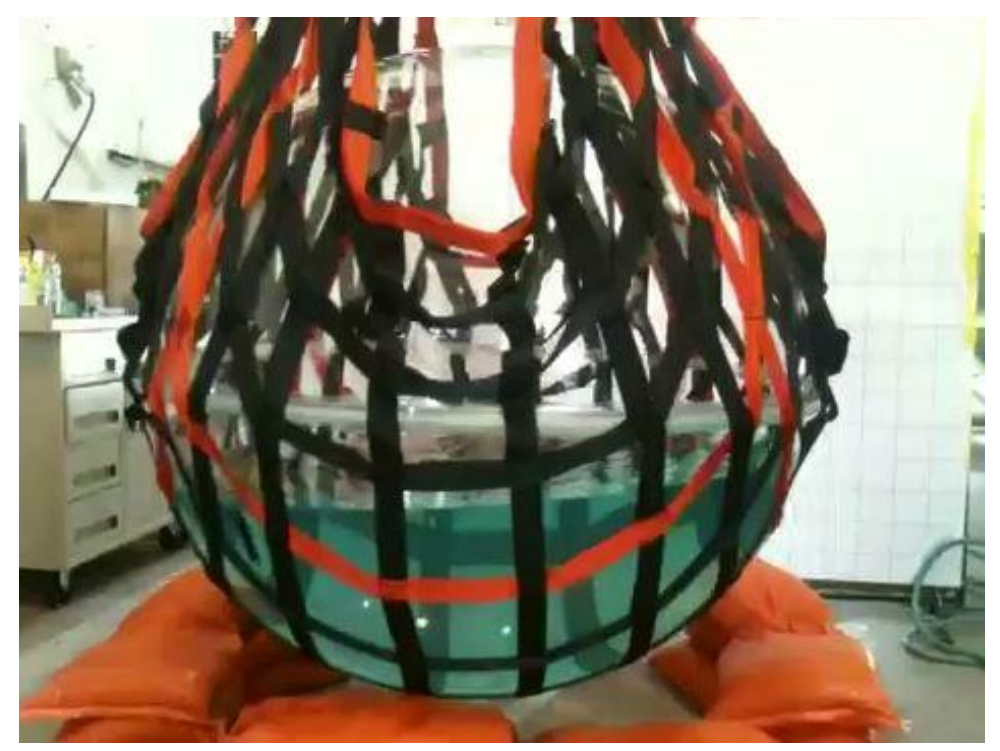

fig-9, full-scale slosh test configuration

As before, the sphere was tested with lateral excitations near the slosh frequency, but also relying on visual feedback of when a 'clean' oscillating slosh wave was being generated. A clean wave could be characterized as a relatively smooth flat surface where one half of the fluid was above the fill level while the other half below. The testing was supported by video recording as well as the mounting of an Apple 'I-phone' device, recording data from its 3-axis accelerometer and then e-mailed to a remote computer. Baffles were then added and the test conditions repeated. Fig-10 shows the sphere with baffles installed while fig-11 shows a test point just over $20 \%$ full, where the test article has just been released and already the large slosh wave is observed being broken up into small minimal energy waves across the surface, due to the baffle below. An I-phone recording data is attached to the front of the tank.

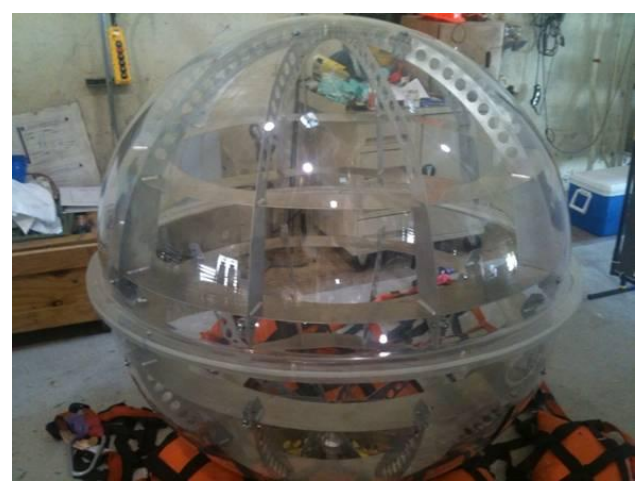

fig-10, Full-scale sphere w/ baffles

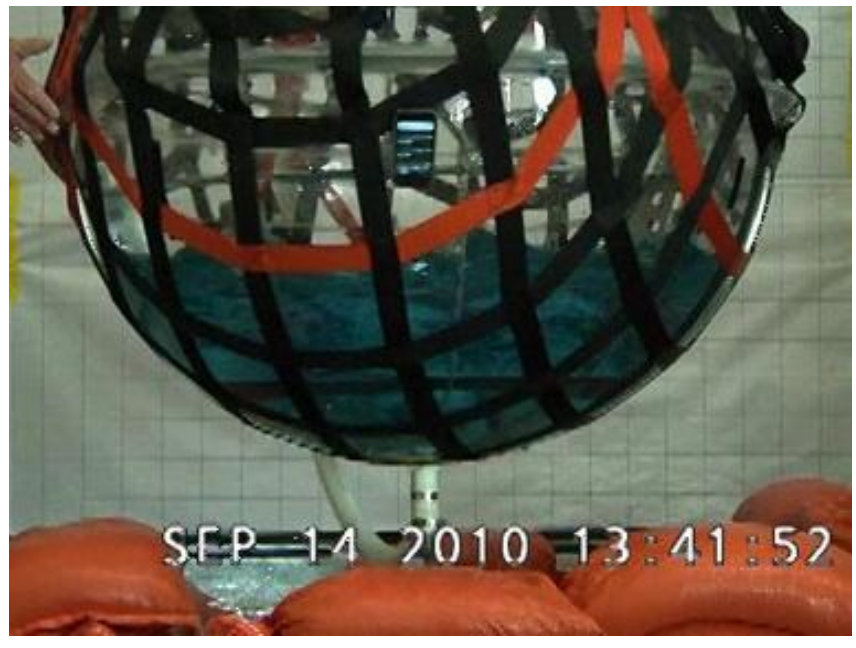

fig-11, Baffles damping slosh at $20 \%$ full 
Table-3 shows both predictions and observed results for slosh frequency and damping at six different fill levels of water within the 48 inch sphere with and without baffles. Damping prediction without baffles is not included for the reasons identified with Pixel testing, the simplified prediction shows trends but is off in magnitude. With baffles frequency data is difficult to observe due to rapid damping, so this data is not included here though theory says it should be the same as without baffles. Frequency generally increased with fill level, as expected, however it remained fairly constant from 33 to 80 $\%$ full, similar to a cylinder. Perhaps the sphere was deforming (though this could not be observed) or perhaps with larger spheres, behavior tends more towards a cylinder for mid fill regions. The observed frequencies, identified both in video and from the I-phone data, also were not any higher than for the 36 inch diameter 'Pixel' test article. It may be that the analytic slosh frequency estimation methodology may begin to diverge for larger tanks. The literature will be reviewed relating to this. As an aside, the I-phone measurements appeared to be generally reasonable and consistent with video observation, except that the I-phone limited data take periods to 8 seconds, and it could not distinguish a good slosh excitation from a poor one (often excited at the wrong frequency or with an overly aggressive amplitude) so a cross-check of the video was still required.

\begin{tabular}{|c|c|c|c|c|c|c|}
\hline \multicolumn{4}{|c|}{ Without Baffles } & \multicolumn{3}{c|}{ With Baffles } \\
\hline Fill level & $\begin{array}{c}\text { Slosh freq } \\
\text { (predict) }\end{array}$ & $\begin{array}{c}\text { Slosh Freq } \\
\text { (observed) }\end{array}$ & $\begin{array}{c}\text { Damping } \\
\text { (observed) }\end{array}$ & Fill level & $\begin{array}{c}\text { Damping } \\
\text { (predict) }\end{array}$ & $\begin{array}{c}\text { Damping } \\
\text { (observed) }\end{array}$ \\
\hline 0.10 & 0.70 & 0.88 & 0.0065 & 0.10 & 0.145 & 0.029 \\
\hline 0.20 & 0.72 & 1.00 & 0.0046 & 0.20 & 0.067 & 0.036 \\
\hline 0.33 & 0.75 & 1.08 & 0.0044 & 0.33 & 0.032 & 0.076 \\
\hline 0.50 & 0.80 & 1.10 & 0.0030 & 0.50 & 0.069 & 0.058 \\
\hline 0.80 & 0.95 & 1.10 & 0.0038 & 0.80 & 0.047 & 0.038 \\
\hline 0.95 & 1.21 & 1.24 & 0.0047 & 0.95 & 0.030 & 0.024 \\
\hline
\end{tabular}

Table-3, full scale slosh predictions vs observed, including baffle effects

Damping results show mostly expected trends and definitely show a strong improvement with the presence of baffles. With the baffles installed, observed damping generally follows predicted (design) trends, except at the low fill levels, where the assumption that the sphere can be approximated locally as a cylinder is less reasonable due to the large change in diameter with small change in height. Otherwise the observed damping ratios are somewhat less than predicted but still mostly over the desired minimum of 0.025 targeted for this design. Fig-12 co-plots the predicted/design 'sawtooth' curve of damping ratios vs observed, (a sawtooth prediction occurs as damping abruptly increases above each baffle). 


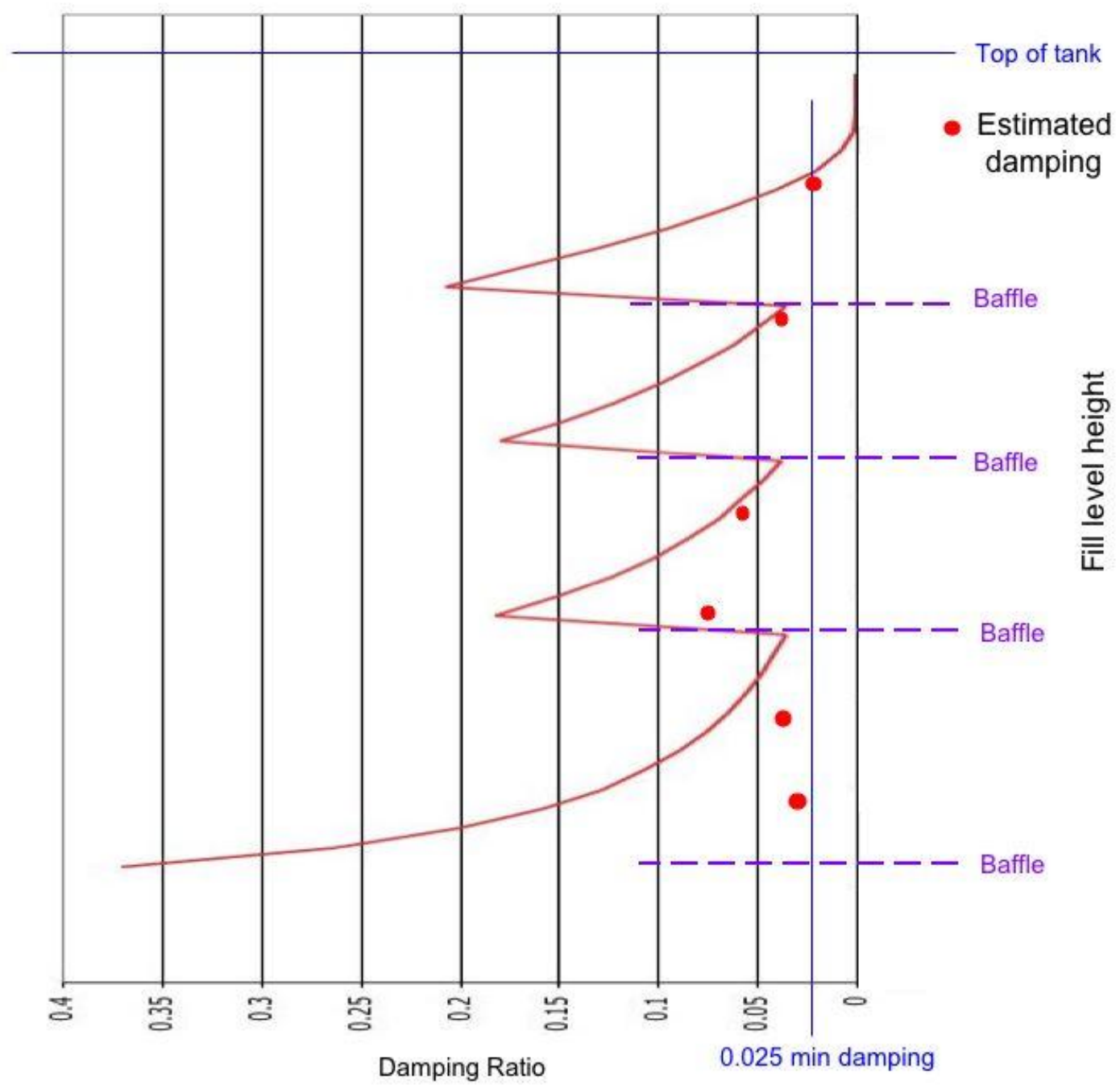

fig-12 predicted damping ratio, w/ baffles, vs observed

\section{Morpheus Testing}

Prior to the actual flight test with the full-scale Morpheus VTB, there was an effort to again characterize full scale multi-tank slosh with cryogenic propellants loaded, essentially repeating the mid-scale 'Pixel' test with the full-scale Morpheus. However this testing would include baffles and in order to isolate any possible differences in fluid damping, a round of slosh testing was performed with only the liquid Methane tanks loaded followed by another round with only the LOX tanks loaded. Other differences were that only one fill level, approximately $20 \%$ was exercised with the methane while 3 levels, 10, 13 and $20 \%$ were exercised with the LOX. Only low propellant loads were used as they are expected to bound loads exercised while flying at the JSC range over the next 12 months. Longer duration flights requiring more propellant are anticipated to occur later at the White Sands Test Facility. 
As with 'Pixel' testing, the Moprheus VTB was suspended from a crane and loaded to the desired propellant level with the appropriate cryogenic. The vehicle was then laterally excited (oscillated) by hand near the expected slosh frequency, then released to oscillate freely and record accelerometer and gyroscopic IMU data, see fig-13. For each round of testing, four different types of input excitations were exercised, including lateral, rocking, impulse and swirl type inputs. Of these, the lateral and impulse inputs appeared to work best for exciting a slosh motion. During the LOX test campaign, excitation of eh empty vehicle was also included to help identify extraneous frequencies and damping due to the rigging as opposed to slosh, so as to isolate these rfom the slosh behavior. Also, it was clearly noted how many excitation cycles occurred at the point of release, to assist in data interpretation.

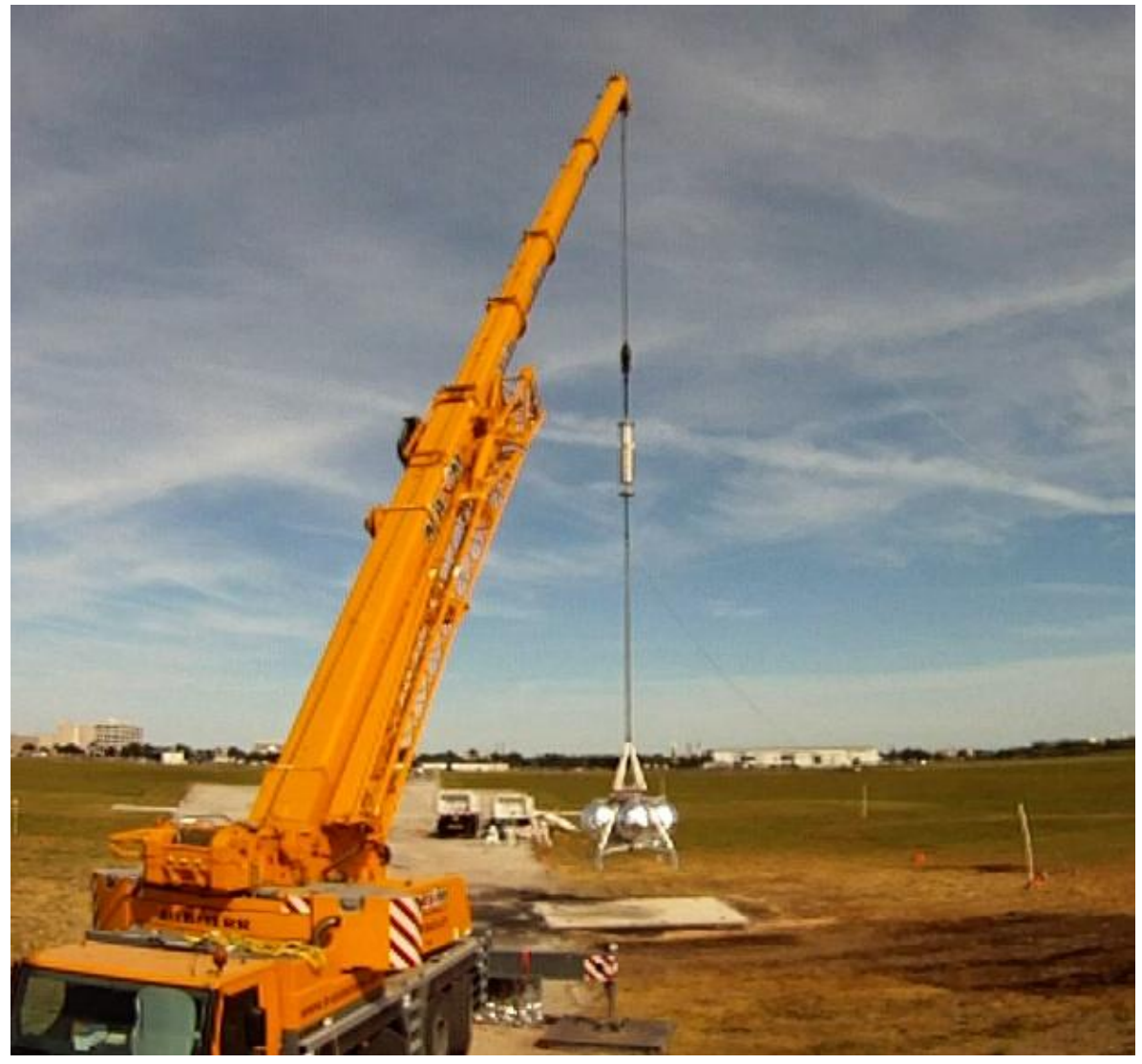

fig-13, Morpheus suspended by crane

\section{Statistical Analysis of Morpheus Slosh Data}

Slosh testing with the full-scale Morpheus lander was done close to the completion deadline of this paper and as such analysis of the full-scale data is incomplete. Still some elements of this on-going process will be covered here to give a flavor for the work. It should be noted that statistical methods are now being employed as several additional frequencies exist in the test set-up and rigging once the test article begins to shake. It was initially believed that these would be easily separable from the slosh frequencies, with 
structural modes of the vehicle being too high, and other pendulum modes, for a pivot point at the crane tip and another at the mounting confluence above the vehicle, being generally quite low (from about 0.35 to $0.1 \mathrm{~Hz}$ ). However, other frequencies appear to be manifesting themselves closer to the slosh frequency, with one suspected to involve the oscillation of the crane tip itself. As such Power Spectral Density (PSD) tools, along with related tools that calculate damping of identified frequencies overlaid in an oscillatory profile, are being explored. [7] Some sample results from an analysis of two lateral excitations at the $10 \%$ fill level during the LOX slosh test, are included here.

The first excitation is captured in fig-14 with a plot of lateral Y-axis acceleration (cross-axis to the LOX tanks) experiencing a Y-axis input over 7 cycles, then free to oscillate. The second excitation is captured in fig-15 with a plot of $\mathrm{Z}$-axis acceleration (axis of LOX tanks) experiencing a Z-axis input over 10 cycles, then free to oscillate. The

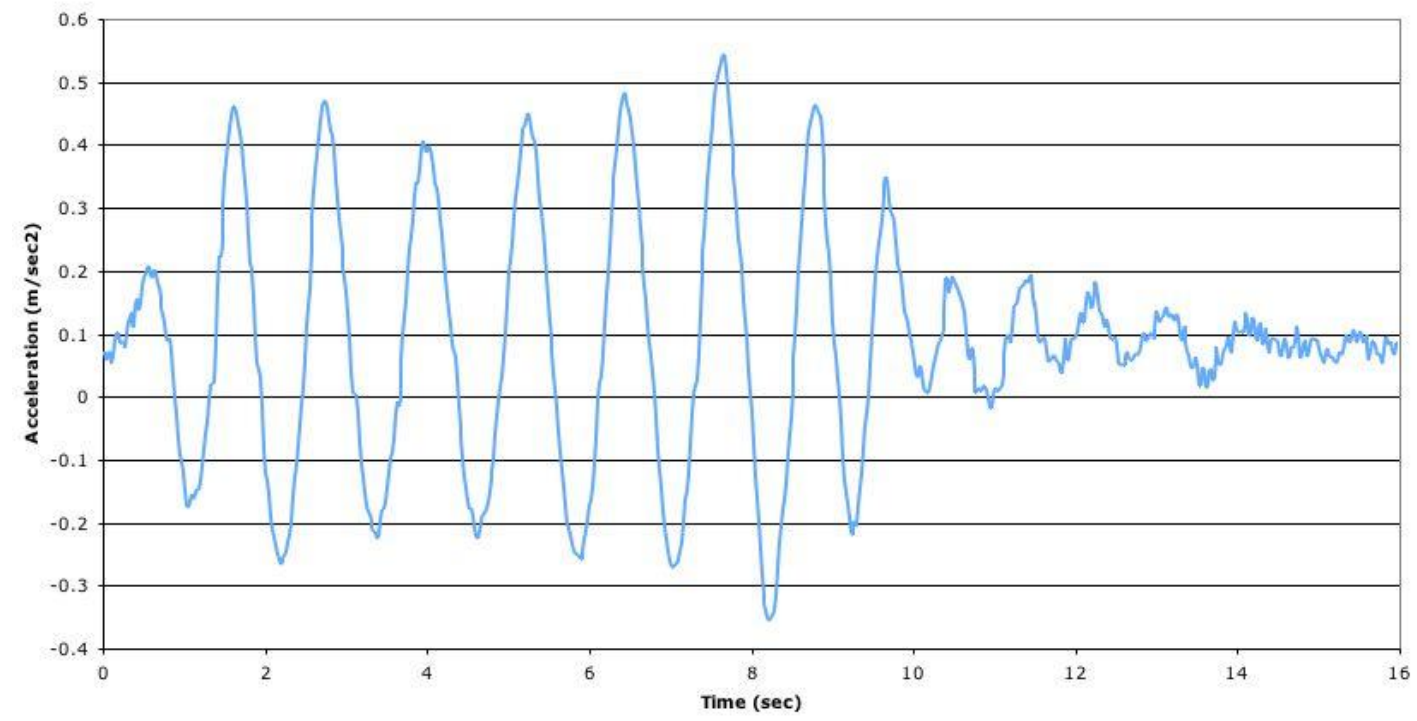

fig-14, Y-axis oscillation w/ LOX tanks $10 \%$ full

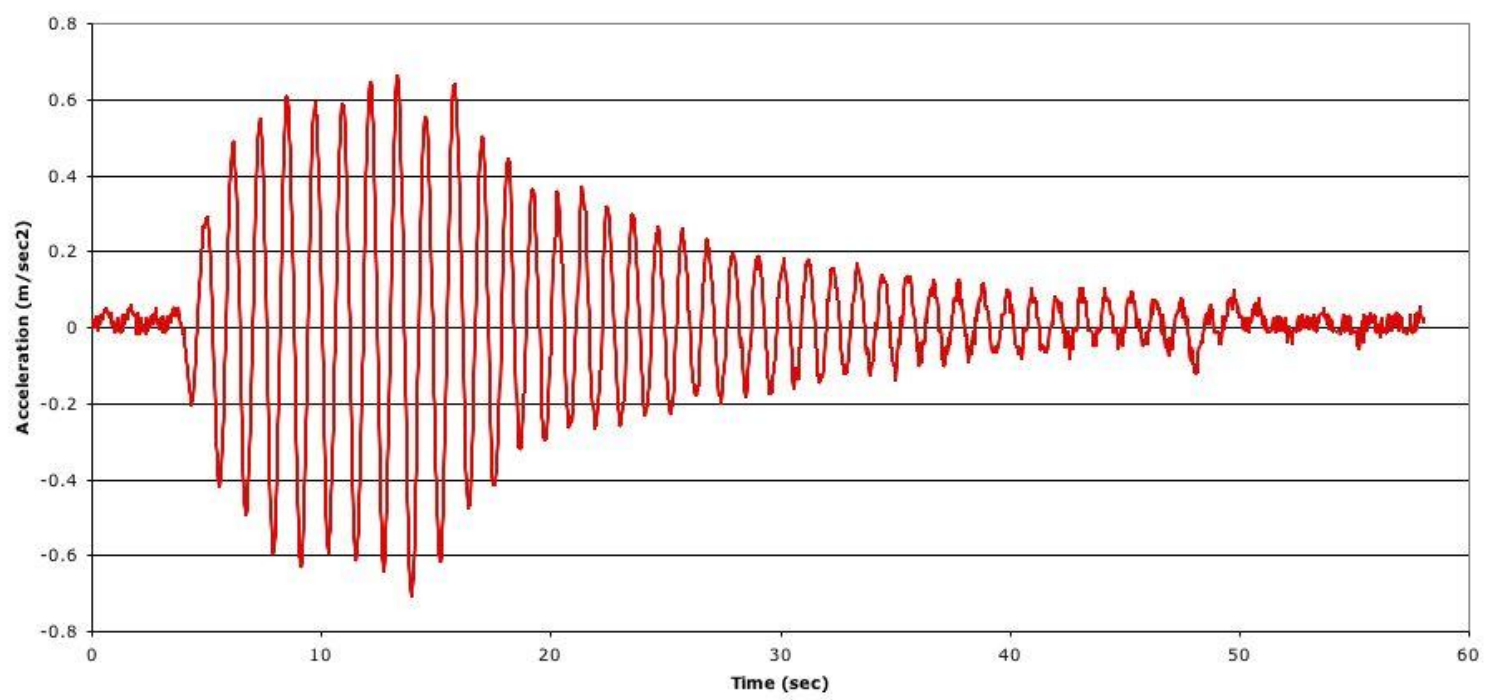

fig-15, Z-axis oscillation w/ LOX tanks $10 \%$ full 
difference in free oscillation time of these two plots is at least partly due to more energy being imparted for the second case (+/- $0.6 \mathrm{~m} / \mathrm{sec} 2$ vs +0.5 to $-0.3 \mathrm{~m} / \mathrm{sec} 2$ excitation), a variation that can occur with human excitation of the slosh mode.

When processing this data, a Power Spectral Density plot was generated for each profile, followed by a statistical estimate of damping for significant frequencies, with results listed in table-4. [7] The suspected slosh frequencies are hi-lighted though further analysis is on-going. The two high frequencies, near $100 \mathrm{hz}$ each, are likely structural frequencies, as they are orders of magnitude too high for slosh. For the Y-axis

\begin{tabular}{|c|c|c|c|}
\hline \multicolumn{2}{|c|}{ Y-axis excitation, 10 \% LOX fill } & \multicolumn{2}{c|}{ Z-axis excitation, 10 \% LOX fill } \\
\hline Freq (hz) & Damping Ratio (\%) & Freq (hz) & Damping Ratio (\%) \\
\hline 101.175 & 0.001 & 99.079 & 0.0 \\
\hline 0.855 & 0.292 & 0.913 & 0.032 \\
\hline 0.926 & 0.047 & 0.917 & 0.0006 \\
\hline 1.142 & 0.207 & 0.839 & 0.102 \\
\hline & & 0.609 & 0.2354 \\
\hline
\end{tabular}

Table-4, estimated slosh damping for the Morpheus LOX tanks at $10 \%$ fill

excitation, the other two frequencies, 0.85 and 1.14 have estimated damping much higher than expected, where amplitude of the oscillation would be cut in-half in less than half a cycle and rapidly diminishing to nothing at all, substantially better damping than even the baffles are expected to provide, and could represent the test article hitting an abrupt, though subtle snag, perhaps a propellant hose, that slows its motion for just an instant. Similarly, the 0.83 and $0.61 \mathrm{hz}$ identified with the Z-axis are experiencing extremely rapid damping, also substantially higher than expected by the baffles. The 0.917 is close to the slosh frequency expected for this condition, however the damping is very low, representing an oscillation that would last for over 130 cycles before decaying to half amplitude, which is substantially less damping than would be expected even without baffles, based on the Pixel test observations. This leaves the 0.92 and 0.913 frequencies, very close together and close to the 0.88 to 0.9 expected for this fill level based on the 48 inch tank study, with their damping at 0.047 and 0.032 respectively, also in the vicinity of what was expected for this fill level, again based on the 48-inch tank study.

\section{Conclusion}

The nature of slosh in spherical tanks, both singularly and in 4-tank clusters in a cruciform pattern, has been explored, as it relates to stability and potential flight control interaction for the Morpheus VTB. This exploration has drawn both from analytic modeling methods developed in the early days large scale rocket flight, as well as from empirical and observational testing with sub-scale, mid-scale and full-scale test articles. The results so far have lead the Morpheus Program to integrate ring baffles in each of its four propellant tanks to mitigate what has been identified as a real potential for detrimental slosh interaction with flight control during terrestrial VTB flights. The baffles themselves were designed based on simple analytic baffle design methods also developed in the 1960's. However this design been tested various times in sub-scale and 
full-scale spherical tank configurations to confirm the desired fluid slosh damping effects, demonstrating an overall acceptable performance from these baffles so far. While investigation is still on-going to interpret the results of the Morpheus slosh tests, results to date, incorporating the use of statistical analysis tools also show promise that the damping provided by this baffle design is adequate, adding confidence that the Morpheus VTB can proceed to its various free flight objectives, with increasing complexity in trajectory shape and aggressive maneuvering.

\section{References}

[1] Abramson, Dr. N. H., "The Dynamic Behavior of Liquids in Moving Containers," NASA SP-106, 1966

[2] Dodge, F. T., "The New "Dynamic Behavior of Liquids in Moving Containers," Southwest Research Institute, San Antonio, Texas, 2000

[3] Lee, A. Y., Strahan, A., Tanimoto, R., and Casillas, A., "Preliminary Characterization of the Altair Lunar Lander Slosh Dynamics and Some Implications for the Thrust Vector Control Design", Proceedings of the AIAA Guidance, Navigation, and Control

Conference, and Exhibit, Toronto, Ontario, Canada, August 2-5, 2010.

[4] Wikipedia entry on Pendulum (mathematics):

http://en.wikipedia.org/wiki/Pendulum_(mathematics)

[5] Anon,: "Slosh Suppression. NASA Space Vehicle Design Criteria (Structures)", NASA SP-8031, 1969.

[6] Wikipedia entry on Damping ratio: http://en.wikipedia.org/wiki/Damping_ratio

[7] reference to be added on statistical tools 